\title{
Is interlimb coordination during walking preserved in children with cerebral palsy?
}

\author{
Pieter Meyns ${ }^{\mathrm{a}, *}$, Leen Van Gestel ${ }^{\mathrm{b}}$, Sjoerd M. Bruijn ${ }^{\mathrm{a}}$, Kaat Desloovere ${ }^{\mathrm{b}, \mathrm{c}}$, \\ Stephan P. Swinnen ${ }^{\mathrm{b}}$, Jacques Duysens ${ }^{\mathrm{a}, \mathrm{d}}$ \\ ${ }^{a}$ Department of Kinesiology, Faculty of Kinesiology and Rehabilitation Sciences, KU Leuven, Heverlee, Belgium \\ ${ }^{\mathrm{b}}$ Department of Rehabilitation Sciences, Faculty of Kinesiology and Rehabilitation Sciences, KU Leuven, Heverlee, Belgium \\ ${ }^{\mathrm{c}}$ Clinical Motion Analysis Laboratory, CERM, University Hospital Leuven, Leuven, Belgium \\ ${ }^{\mathrm{d}}$ Department of Research, Development and Education, Sint Maartenskliniek, Nijmegen, The Netherlands
}

\section{A R T I C L E I N F O}

Article history:

Received 19 December 2011

Received in revised form 5 March 2012

Accepted 6 March 2012

Available online 21 April 2012

\section{Keywords:}

Cerebral palsy

Gait

Interlimb Coordination

Arm swing

Walking speed

Speed increment

\begin{abstract}
A B S T R A C T
Arm movements during gait in children with cerebral palsy (CP) are altered compared to typically developing children (TD). We investigated whether these changes in arm movements alter interlimb coordination in $\mathrm{CP}$ gait. 3D gait analysis was performed in $\mathrm{CP}$ (diplegia [DI]: $N=15$ and hemiplegia [HE]: $N=11)$ and TD $(N=24)$ children at preferred and fast walking speeds. Mean Relative Phase (MRP, i.e. mean over the gait cycle of the Continuous Relative Phase or (RP) was calculated as a measure of coordination, standard deviation of CRP was used as a measure of coordinative stability, and the sign of MRP indicated which limb was leading (for all pair combinations of the four limbs). In HE, coordination was significantly altered, less stable and a different leading limb was found compared to TD whenever the most affected arm was included in the studied limb pair. In DI, coordination deteriorated significantly when any of the two legs was included in the studied limb pair, and coordinative stability was significantly affected when any of the two arms was included. In almost all limb pair combinations, a different limb was leading in DI compared to TD. Increasing walking speed significantly improved coordination and coordinative stability of several limb pairs in DI. Coordination and limb-leading deficits were mostly linked to the affected limb. The compensating (non-affected) arm primarily affected coordinative stability, which underlines the importance of active arm movements in HE. Increasing walking speed may be used to improve interlimb coordination in DI.
\end{abstract}

(c) 2012 Elsevier Ltd. All rights reserved.

\section{Introduction}

Cerebral palsy $(\mathrm{CP})$ is a developmental disorder resulting from injury to the central nervous system in the immature brain (Aisen et al., 2011), often leading to persistent abnormal limb strength, muscle spasticity and motor control deficits. Different types of cerebral palsy are based on the topography of the affected limbs (Dabney, Lipton, \& Miller, 1997). In children with hemiplegia (HE) one side of the body is affected while in children with diplegia (DI) the lower limbs are affected more than the upper limbs (Dabney et al., 1997).

\footnotetext{
* Corresponding author at: Department of Kinesiology, KU Leuven, Tervuursevest 101, Box 1501, B-3001 Heverlee, Belgium. Tel.: +32 16329065; fax: +32 16329197.

E-mail addresses: pieter.meyns@gmail.com, Pieter.meyns@faber.kuleuven.be (P. Meyns).
} 
A fundamental part of the rehabilitation strategy in children with $\mathrm{CP}$ is focused on sustaining or improving independent locomotion. The use of arm movements during gait seems to have a potential beneficial influence on gait rehabilitation. In patients with spinal cord injury, for instance, it was found that the natural reciprocating arm swing facilitated stepping. On the contrary, weight bearing on the arms inhibited rhythmic stepping (Behrman \& Harkema, 2000). Additionally, the use of sliding handles on handrails that allows for reciprocating arm swing during walking, yielded promising effects for the gait rehabilitation in stroke patients (Stephenson, De Serres, \& Lamontagne, 2010; Stephenson, Lamontagne, \& De Serres, 2009). Moreover, even in healthy participants, the use of arm movements has been found to improve functional mobility test scores compared to when arm movements were not allowed (Milosevic, McConville, \& Masani, 2011). In children with CP, the use of arm movements has not received much attention for this purpose.

However, we recently demonstrated that arm swing during gait in CP children is altered compared to typically developing (TD) children (Meyns et al., 2011). HE children exhibited a smaller swing of the hemiplegic arm compared to TD children. Such a smaller swing could be due to weakness, spasticity and/or deficient motor control (Rose, 2009). Additionally, we reported that the arm swing amplitude on the non-hemiplegic side was significantly increased (Meyns et al., 2011), both when compared to TD children and to the unaffected arm. The latter finding has been suggested to be a compensatory strategy to control total body angular momentum (Bruijn, Meyns, Jonkers, Kaat, \& Duysens, 2011). DI children, on the other hand, do not show this asymmetry in arm swing during walking, and they are able to increase arm swing amplitude with increasing walking speed (in contrast to HE children) (Meyns et al., 2011). However, DI children show increased bilateral shoulder abduction and elbow flexion (Romkes et al., 2007) and hold their hands more elevated and anterior compared to TD children during walking (Meyns et al., 2012). These arm postures are thought to be a compensation strategy to maintain stability during walking (Meyns et al., 2012).

It is expected that these arm swing alterations during gait lead to changed interlimb coordination. Alterations in arm swing during gait have been shown to affect interlimb coordination in stroke and Parkinson's disease (Wagenaar \& Van Emmerik, 1994), however, it has not been assessed before in CP. Studying interlimb coordination of gait allows for assessing pathology induced spatio-temporal movement deficits rather than spatial or temporal deficits only, as is the case with traditional measures (Krasovsky \& Levin, 2010). So far, most studies on CP have not gone beyond these traditional spatial and temporal quantifications of gait. Still, it has been shown that coordination measures are an important determinant of gait quality, and they are considered a meaningful goal in rehabilitation (Donker \& Beek, 2002). Therefore, in the current study, we aimed to investigate interlimb coordination during walking in $\mathrm{CP}$ in order to assess the movement deficits of this group in an integrated manner, combining all limbs and spatial and temporal characteristics of the movements.

When studying interlimb coordination, the frequency ratio between arm movements and leg movements is often described as well. At preferred walking speeds, one arm swing is typically associated with one leg swing (i.e. 1:1 arm-to-leg swing ratio). At low walking speeds, however, a 2:1 frequency ratio with respect to the leg movements has been reported (Van Emmerik, Wagenaar, \& Van Wegen, 1998; Wagenaar \& Van Emmerik, 2000). At higher walking speeds, healthy adults abandon the 2:1 arm-to-leg ratio, and adopt a 1:1 arm-to-leg ratio. In subjects with central neurological deficits (e.g. stroke and Parkinson's disease), however, this 2:1 arm-to-leg swing ratio persists at higher walking speeds (Wagenaar \& Van Emmerik, 1994). Interestingly, in stroke patients the hemiplegic arm shows the 2:1 arm-to-leg swing ratio, while the nonhemiplegic arm shows a 1:1 arm-to-leg swing ratio (Wagenaar \& Van Emmerik, 1994).

Changes in interlimb coordination could potentially be accompanied by an alteration of the limb that is leading the movement (i.e. leading limb) and the limb that is following this leading limb (i.e. lagging limb). Such alterations in the leading limb have already been described in patients with Parkinson's disease (Nanhoe-Mahabier et al., 2011). In an interlimb cycling study, it was shown that arm cycling cadence was altered when leg cycling cadence was changed but not vice versa (Sakamoto et al., 2007). This indicates that the legs lead the arms under these conditions. Whether this is also true for gait remains to be investigated, even in healthy subjects. However, it could be that in CP patients (especially DI children), where the legs are more affected, arm movements play a more prominent role, and thereby lead the leg movements.

Since interlimb coordination has not been studied before in $\mathrm{CP}$ gait, the aim of the current study was first to investigate whether interlimb coordination is altered in CP gait compared to TD children. In doing so, we focused on arm-to-leg swing ratio and the leading of limbs. Since walking speed influences arm swing differently in HE and DI children, we studied interlimb coordination for two walking speeds.

\section{Material and methods}

\subsection{Participants}

The same 26 CP (4-12 y) and 24 TD children (5-12 y) from a previous study (Meyns et al., 2011) were included in the current study. The CP group consisted of $11 \mathrm{HE}$ children and 15 DI children. Participant characteristics are presented in Table 1. CP children were recruited from the Laboratory of Clinical Movement Analysis of the University Hospital Pellenberg (U.Z. Leuven) and included if they (1) were able to walk without walking aids (based on the Gross Motor Function Classification System or GMFCS), (2) were diagnosed with the predominantly spastic type of CP, (3) had sufficient cooperation to follow verbal instructions, (4) did not undergo Botulinum Toxin A treatment within the past 6 months or (5) did not previously receive lower limb surgery. The GMFCS is a five level classification system that describes the gross motor function of children with CP on the basis of their self-initiated movement with particular emphasis on sitting, walking, and wheeled mobility. 
Table 1

Characteristics of TD children and children with CP.

\begin{tabular}{|c|c|c|c|c|c|}
\hline & TD & Diplegia & Hemiplegia & Test statistic & $p$ \\
\hline$N$ & 24 & 15 & 11 & - & - \\
\hline Ethnicity (caucasoid/negroid) & $24 / 0$ & $15 / 0$ & $10 / 1$ & $\chi_{[2, N=50]}^{2}=3.62$ & 0.16 \\
\hline Gender $(\mathrm{M} / \mathrm{F})$ & $12 / 12$ & $11 / 4$ & $8 / 3$ & $\chi_{[2, N=50]}^{2}=2.82$ & 0.24 \\
\hline GMFCS (I/II/III) & - & $8 / 6 / 1^{a}$ & $7 / 4 / 0$ & $\chi_{[2, N=26]}^{2}=0.87$ & 0.65 \\
\hline Age (y: years, m: months) & 9 y 4 m (2y $1 \mathrm{~m})$ & 9 y $11 \mathrm{~m}(2$ y $5 \mathrm{~m})$ & 7 y 9 m (2 y 11 m) & $F_{[2,47]}=2.52$ & 0.09 \\
\hline Weight $(\mathrm{kg})$ & $31.72(8.64)$ & $31.54(13.36)$ & $23.87(7.57)$ & $F_{[2,47]}=2.55$ & 0.09 \\
\hline Height (m) & $1.38(0.14)$ & $1.34(0.19)$ & $1.22(0.15)^{\mathrm{b}}$ & $F_{[2,47]}=3.69$ & 0.03 \\
\hline Speed FW (m/s) & $1.20(0.16)$ & $0.94(0.24)^{\mathrm{c}}$ & $1.10(0.13)$ & $F_{[2,46]}=11.45$ & $<\mathbf{0 . 0 0 1}$ \\
\hline Speed FFW $(\mathrm{m} / \mathrm{s})$ & $1.95(0.17)$ & $1.41(0.41)^{\mathrm{c}}$ & $1.67(0.18)^{c}$ & $F_{[2,46]}=11.45$ & $<\mathbf{0 . 0 0 1}$ \\
\hline
\end{tabular}

Modified from Meyns et al. (2011).

Note that age, weight, height and speed are presented as follows: mean (standard deviation). $N=$ number of subjects, $\mathrm{M} / \mathrm{F}=\mathrm{male} / \mathrm{female}, \mathrm{GMFCS}=\mathrm{Gross}$ Motor Function Classification System, FW = preferred walking speed condition, FFW = as fast as possible walking speed condition. Differences that were significant are presented in bold.

a 1 subject with a GMFCS level III was included because this subject was able to complete the walking trials of the experiment without walking aids.

b Significantly different from TD (one way Analysis of Variance, post hoc Tukey's HSD: TD vs DI, $p=0.025$ )

c Significantly different from TD (repeated measures ANOVA: post hoc Tukey's HSD: TD-FW vs DI-FW, $p=0.013$; TD-FFW vs DI-FFW, $p<0.001$; TD-FFW vs HE-FFW, $p=0.007$ ).

Persons with a GMCFS level of I to III can achieve self-mobility without using a powered wheelchair (Palisano et al., 1997). Diagnosis and type of CP was determined by a multidisciplinary team of neuropediatricians, pediatric orthopedicians and rehabilitation medicine physicians, and was based on neuropediatric and neurologic examinations including medical imaging (i.e. magnetic resonance imaging). All experiments were approved by the local ethical committee and were performed with the informed, written consent of the participants' parents in accordance with the Declaration of Helsinki.

\subsection{Protocol}

An eight camera Vicon system (Oxford Metrics, Oxford, UK) was used to record three-dimensional total-body kinematic data (100 samples/s) with the PlugInGait markerset (Davis, Ounpuu, Tyburski, \& Gage, 1991). A total of 34 retro-reflective markers were positioned on the skin overlying specific bony landmarks or anatomical positions of the children's upper and lower body. The lower body marker set corresponded to the Helen Hayes Marker set described by Kadaba, Ramakrishnan, and Wootten (1990). The upper body marker set has been described previously by Gutierrez, Bartonek, Haglund-Akerlind, and Saraste (2003). The upper body marker set was always placed by the same researcher. The lower body marker set was always placed by a physical therapist of the University hospital especially trained for clinical gait analysis. Participants walked at a preferred (FW) and "as fast as possible" (without running; FFW) speed along a 10-m walkway, aiming for three successful trials at each speed. Successful trials included four consecutive foot strikes with full-marker-visibility. Trials were not retained when the participant made excessive movements of the head, arms or trunk unrelated to walking. Participants were granted some practice trials before each condition. Despite careful measurement, some trials had to be omitted due to irregularities in the kinematics (five for TD, one for $\mathrm{DI}$ ).

\subsection{Data processing}

Marker coordinates were filtered and smoothed using Woltring's quintic spline routine (Woltring, 1986). Vicon Workstation and Polygon software (Oxford Metrics, Oxford, UK) were used to define gait cycles and estimate internal joint centres. The most affected side in CP was determined as the side on which the leg had the highest spasticity measures (i.e. mean of the Modified Ashworth Scale values for the hip adductors, hip flexors, hamstrings, gastrocnemius, soleus and tibialis posterior). For TD, the least affected side was defined as their dominant side.

Time-courses of angular displacement and angular velocity of upper arms and upper legs with respect to the vertical (positive forward) were computed in the sagittal plane (i.e. elevation angles; see Figs. 1 and 2). All angular profiles were normalized in time (to a percentage of the stride duration) and amplitude (such that maximum and minimum were 1 and -1, respectively). From these profiles, trials in which patients did not maintain a 1:1 arm-to-leg swing ratio (i.e. arms showed more oscillations than legs) were identified, and excluded from further relative phase analysis.

Phase angles (PA) of each segment were calculated from the normalized angular profiles and their derivatives. A PA was determined on the phase portrait of a limbs movement (the phase portrait of a limb is a graph with the angular displacement of the limb on the $\mathrm{x}$-axis and the angular velocity of the limb on the $\mathrm{y}$-axis). The phase portrait trajectory was transformed from Cartesian to polar coordinates. The angle formed by the radius and the horizontal axis was the PA of the trajectory. These are needed to calculate the relative phase between two limbs. A detailed description of the calculation of PA can be found in Stergiou, 2004. Next, the continuous relative phase (CRP; see Fig. 2) between the different segments was calculated by subtracting the appropriate limb PA time series from each other (Stergiou, 2004). CRP between a leg and arm segment was calculated as leg PA minus arm PA (positive values indicating leading of the leg). CRP between arms or legs was determined 


$$
\overline{-1 \cdot} \operatorname{arm}(\alpha)
$$
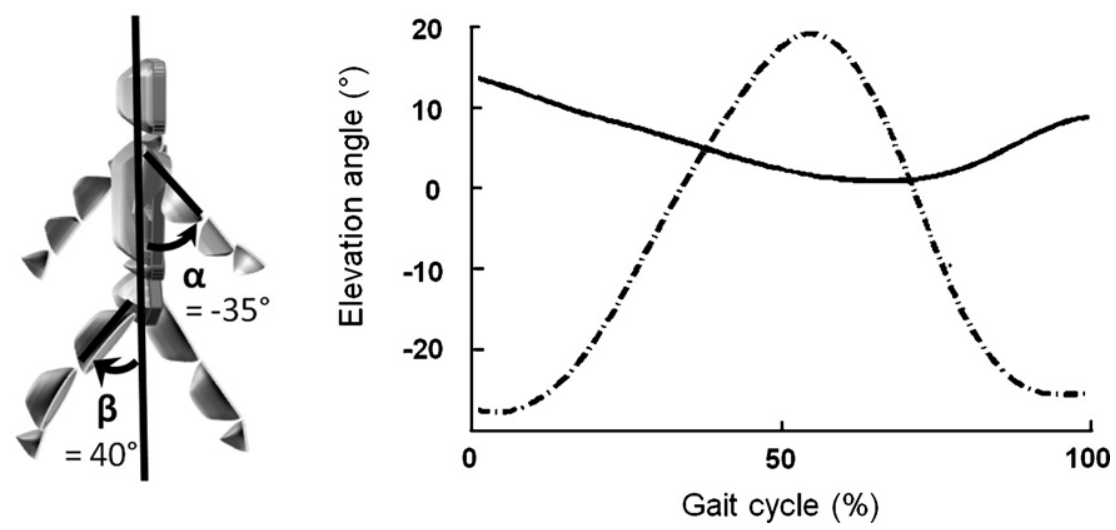

Fig. 1. Elevation angles. (Left) Schematic representation of the angular displacement of the upper arm and upper leg segment with respect to the vertical (i.e. elevation angle). (Right) example of the elevation angles (of the upper arm and leg) for one gait cycle in a typically developing child (TD).

as least affected limb PA minus most affected limb PA (positive values indicating leading of the least affected limb). A relative phase of $0^{\circ}$ reflected that two segments were perfectly in-phase (i.e. moving in the same direction at the same time or synchronized), while a relative phase of $180^{\circ}$ reflected they were in anti-phase (i.e. moving in opposite directions at the same time or alternated).

From these CRP profiles, three measures were extracted. First, mean absolute CRP over the gait cycle (MARP) was used to quantify the coordination between limbs. Thus, MARP is a measure of how the movements of two segments are timed with respect to each other. Second, the standard deviation of the CRP over the gait cycle (SD of CRP) was calculated as a measure for coordinative stability (Donker \& Beek, 2002; Meesen, Wenderoth, Temprado, Summers, \& Swinnen, 2006; Swinnen, Jardin, Meulenbroek, Dounskaia, \& Hofkens-Van Den Brandt, 1997). Finally, the sign of the mean CRP (MRP) over the gait cycle was calculated to indicate which limb was leading the movement and which limb was following. These measures provide information about the coordination between two segments. Therefore, the measures of coordination were calculated between all pair combinations of the upper arm and upper leg segments. To avoid cumbersome repetitions of each limb pair combination in the results section, the limb pairs have been abbreviated when a measure of coordination was described (in parentheses; i.e. least affected arm-least affected leg: LALL; most affected arm-most affected leg: MAML; least affected arm-most affected leg: LAML; most affected arm-least affected leg: MALL). All measures were calculated using circular statistics (Fisher, 1993).

\subsection{Statistical analysis}

Data normality was checked using Kolmogorov-Smirnov and Shapiro-Wilk $W$ tests (see Supplementary data 1).

Categorical demographic variables (i.e. ethnicity, gender, GMFCS level) were compared using Chi-square tests. Continuous demographic variables (i.e. age, weight, height) were compared using a one-way ANOVAs. Effects of Group and Condition on walking speed were tested using a repeated measures ANOVA.

Next, Chi-square tests were applied to determine whether the number of participants showing 1:1 arm-to-leg swing ratio trials differed between groups. For these Chi-square tests all (successful) trials were analyzed as separate trials, whereas for statistics on interlimb coordination measures the average over the three trials (per condition) was used.

Effects of Group, Speed and Group * Speed on interlimb coordination measures (except limb-leading) were tested using Generalized Estimation Equations (Liang \& Zeger, 1986; Zeger \& Liang, 1986). This allowed for using actual measured walking speed (in both conditions) as a continuous rather than categorical variable. Whenever an interaction did not reach significance, it was removed from the model. Chi-square tests were applied to test for differences between the number of participants who showed a leading or a lagging limb. SPSS 16.0.2 was used for statistical analysis, and $\alpha=0.05$ was used to establish statistical significance.

\section{Results}

\subsection{Arm-to-leg ratio}

For TD the percentage of 1:1 trials was 95.6\% (135/141), while for DI it was 78.1\% (73/89) and for HE it was 75.5\% (53/66). These differences between the CP groups and TD were statistically significant, $\chi_{[2, N=296]}^{2}=14.903, p<0.001$. 


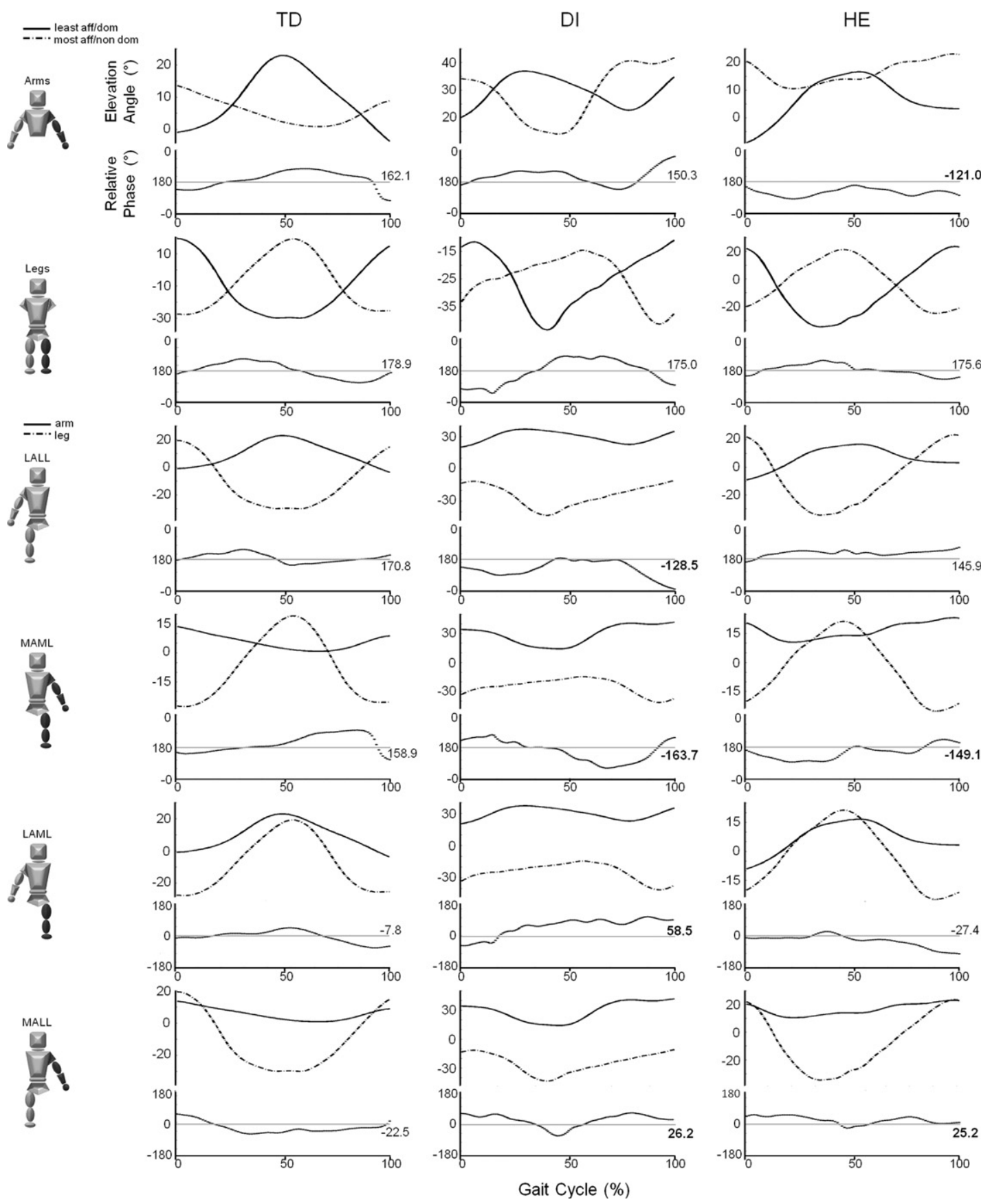

Fig. 2. Typical examples of the elevation angles and the continuous relative phase. The angular displacement with respect to the vertical (i.e. elevation angle) of the upper arm and leg segments and the continuous relative phase (CRP) of the six limb pair combinations are shown for one gait cycle in a typical developing child (TD, left column), a child with diplegia (DI, middle column) and a child with hemiplegia (HE, right column). The MRP (Mean Relative Phase) values of the different limb pairs are presented on the right of the CRP plot. Abbreviations: $L A L L=$ least affected arm-least affected leg, MAML $=$ most affected arm-most affected leg, LAML = least affected arm-most affected leg, MALL = most affected arm-least affected leg. In this example, LALL is more coordinated in the TD child compared to both CP children. Since these two limbs in the TD child are moving more adequately in the opposite direction at the same time, the MRP value also approached $180^{\circ}$ more closely. From the CRP plot it can be derived whether the MRP will be positive or negative (e.g. when most of the curve is positive, the MRP will be positive as well). The sign of the MRP indicates which limb is leading the other. A positive relative phase value indicates that the leg leads the arm segment (or the least affected limb leads the most affected limb). In case of the LALL for the TD child, $170.8^{\circ}$ means that the LALL are coordinated in an out-of-phase pattern but the leg is leading the movement. Note that the MRP values of the CP children with a different sign than the TD child are depicted in bold. 
In HE, the 13 trials ( 6 in FW [preferred walking speed], 7 in FFW [fast walking speed]) with a different arm-to-leg ratio than 1:1, were distributed over 8 participants. For all but one trial, a 2:1 arm-to-leg ratio was found only on the most affected side, while the least affected arm showed a 1:1 frequency coordination with the legs.

In DI, sixteen 2:1 arm to leg coordination trials (11 in FW, 5 in FFW) were distributed over 8 participants. In DI, for most trials (10) it was the most affected arm that swung at a higher frequency while the least affected arm had a 1:1 arm-to-leg swing ratio. For 4 trials the opposite was the case, while for the remaining 2 trials both sides showed a 2:1 swing ratio. This 2:1 arm-to-leg swing ratio (5 in FW, 1 in FFW) was apparent in only 4 TD (on the non-dominant side only).

\subsection{Mean absolute relative phase}

Differences in mean absolute relative phase (MARP) between groups were found for all limb pair combinations (Fig. 3).

In HE, arms (Fig. 3, left) moved less in anti-phase (i.e. alternation) than in TD (TD vs HE: $\chi^{2}[2,43]=6.62, p=0.01$ ). For the coordination between the arms, there was no significant difference between DI and TD (TD vs DI: $\chi^{2}{ }_{[2,43]}=1.62, p=0.204$ ). For both HE and DI, legs (Fig. 3, left) moved less in anti-phase than in TD (TD vs DI: $\chi^{2}{ }_{[2,44]}=11.04, p<0.001$; TD vs HE: $\left.\chi^{2}{ }_{[2,44]}=10.89, p<0.001\right)$.

Considering coordination of the ipsilateral limbs (Fig. 3, middle), both the least (least affected arm-least affected leg, LALL) and most affected (most affected arm-most affected leg, MAML) limb pairs of DI were significantly less in anti-phase than in TD (MARP of LALL: TD vs DI: $\chi^{2}{ }_{[2,44]}=8.03, p=0.005$, TD vs HE: $\chi_{[2,44]}^{2}=0.26, p=0.609$; MAML: TD vs DI: $\chi_{[2,43]}^{2}=5.96, p=0.015$, TD vs HE: $\left.\chi_{[2,43]}^{2}=2.02, p=0.155\right)$. Moreover, DI moved the limbs on the least affected side less in anti-phase than HE $\left(\chi^{2}[2,44]=8.08, p=0.004\right)$. Additionally, for both most and least affected sides a main effect of Speed was found (MARP of LALL: $\chi^{2}{ }_{[1,44]}=4.23, p=0.04$; MAML: $\chi^{2}{ }_{[1,44]}=7.87, p=0.005$ ). A significant Group ${ }^{*}$ Speed interaction effect revealed that when walking speed increased, DI moved ipsilateral limb pairs more toward anti-phase than HE (for least affected side) and TD (for both sides; MARP of LALL: TD vs DI ${ }^{*}$ Speed: $\chi^{2}{ }_{[2,44]}=8.26, p=0.004$, DI vs HE * Speed: $\chi^{2}{ }_{[2,44]}=7.84$, $p=0.005$, TD vs HE ${ }^{*}$ Speed: $\chi^{2}{ }_{[2,44]}=0.16, p=0.693$; MAML: TD vs DI ${ }^{*}$ Speed: $\chi^{2}[2,43]=5.69, p=0.017$, DI vs HE* Speed: $\chi^{2}[2,43]=0.55, p=0.459$, TD vs HE* Speed: $\left.\chi^{2}[2,43]=1.10, p=0.293\right)$.

Considering contralateral arm-leg combinations (Fig. 3, right), the least affected arm and most affected leg (LAML) were significantly less in phase in DI than in HE and TD (DI vs TD: $\chi^{2}{ }_{[2,44]}=7.66, p=0.006$, DI vs HE: $\chi^{2}{ }_{[2,44]}=8.01, p=0.005$, TD vs

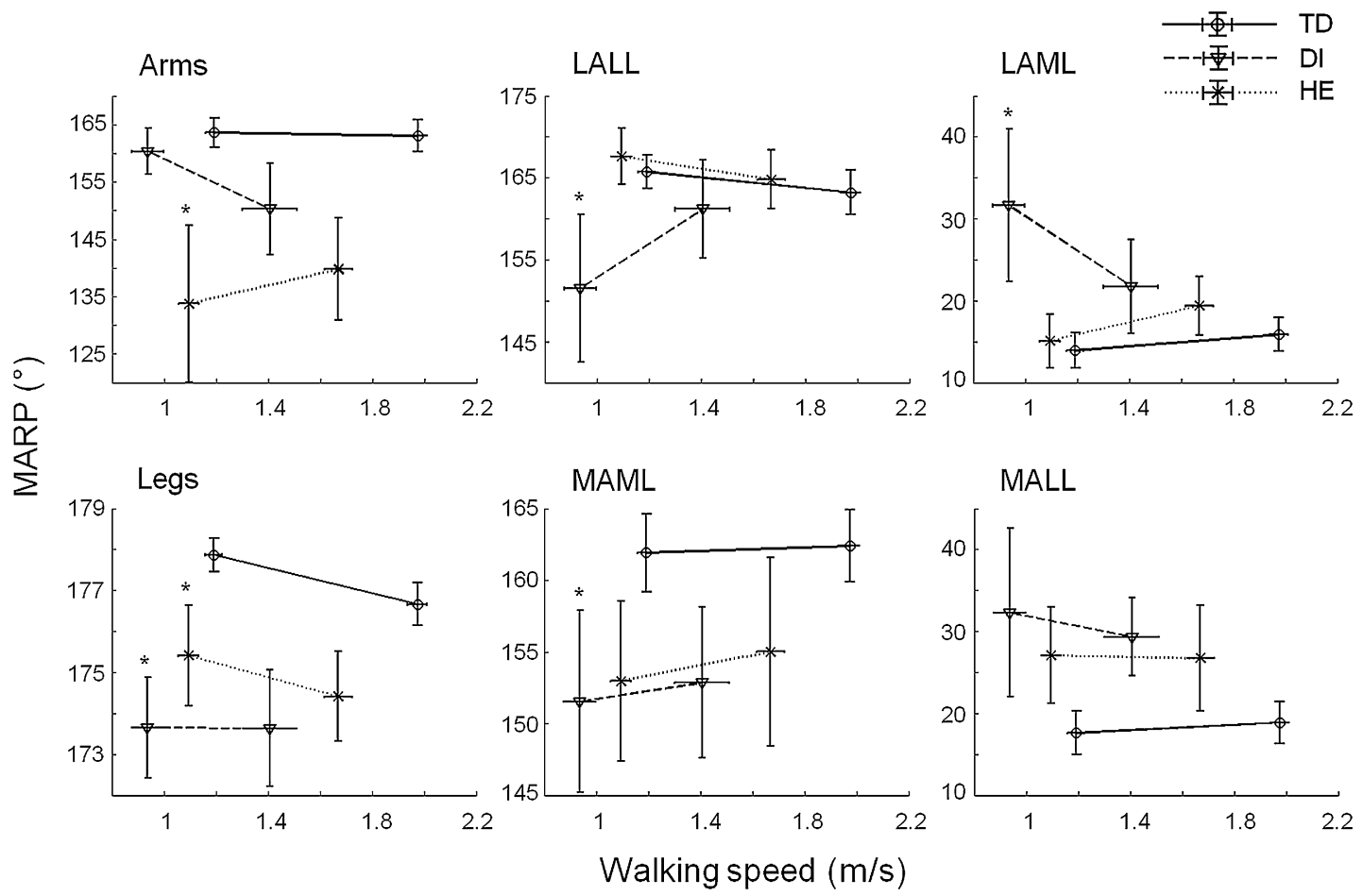

Fig. 3. Comparison of the level of coordination between the three groups. Mean Absolute Relative Phase (MARP) over the gait cycle is presented for the three groups. The symbols representing the groups are rounds for typically developing children (TD), triangles for children with diplegia (DI), and crosses for children with hemiplegia (HE). There are two symbols for each group, one for each walking speed condition. Additionally, the lines connecting the symbols are different for the three groups (full for TD, dashed for DI, and dotted for HE). For homologous (arms, legs) and ipsilateral (LALL, MAML) limb pairs the maximal expected MARP score is $180^{\circ}$ (alternation), whereas the crossed (LAML, MALL) limb pairs are expected to be close to $0^{\circ}$ (synchronization). Error bars represent standard errors of the mean. Abbreviations: LALL = least affected arm-least affected leg, MAML = most affected arm-most affected leg, LAML = least affected arm-most affected leg, MALL = most affected arm-least affected leg. *Significantly different from TD (group effect). 
HE: $\left.\chi_{[2,44]}^{2}=0.20, p=0.651\right)$. This difference appeared to diminish when walking speed increased, as was also evidenced by a significant Group * Speed interaction effect (TD vs DI ${ }^{*}$ Speed: $\chi^{2}{ }_{[2,44]}=6.76, p=0.009$, TD vs HE ${ }^{*}$ Speed: $\chi^{2}{ }_{[2,44]}=0.68$, $p=0.411$ ). There were no significant differences in coordination between the most affected arm and least affected leg (MALL) between groups (TD vs DI: $\chi^{2}{ }_{[2,43]}=2.80, p=0.094$, TD vs HE: $\chi^{2}{ }_{[2,43]}=2.40, p=0.122$ ).

\subsection{Standard deviation of the CRP}

Standard Deviation of the CRP (SD of CRP) was higher in CP compared to TD (Fig. 4).

Coordination between the arms (Fig. 4, left) was significantly more stable for TD than for both CP groups (TD vs DI: $\chi_{[2,43]}^{2}=12.17, p<0.001$, TD vs HE: $\left.\chi_{[2,43]}^{2}=14.30, p<0.001\right)$. Coordination of the legs, however, (Fig. 4, left) was more stable in HE than in TD and DI (HE vs TD: $\chi_{[2,44]}^{2}=55.39, p<0.001$, HE vs DI: $\chi^{2}{ }_{[2,44]}=14.03, p<0.001$ ).

Considering coordination of the ipsilateral limbs (Fig. 4, middle), coordination between the least affected limbs was less stable in DI compared to HE and TD (DI vs TD: $\chi_{[2,44]}^{2}=11.74, p<0.001$, DI vs HE: $\chi^{2}{ }_{[2,44]}=6.20, p=0.013$, TD vs HE: $\left.\chi_{[2,44]}^{2}=0.20, p=0.654\right)$. For the most affected side, coordination was more stable for TD than for HE (TD vs DI: $\chi^{2}{ }_{[2,43]}=3.51$, $p=0.061$, TD vs HE: $\left.\chi^{2}[2,43]=5.10, p=0.024\right)$.

Considering contralateral arm-leg combinations (Fig. 4, right), coordinative stability in DI of least affected arm and most affected leg was decreased with respect to TD (TD vs DI: $\chi^{2}{ }_{[2,44]}=6.11, p=0.013$, TD vs HE: $\chi^{2}{ }_{[2,44]}=0.00001, p=0.997$ ). Coordination between most affected arm and least affected leg was significantly more stable for TD than for both CP groups (TD vs DI: $\chi_{[2,44]}^{2}=5.97, p=0.011$, TD vs HE: $\chi_{[2,44]}^{2}=8.10, p<0.001$ ).

A main effect of Speed on the variability of the CRP for almost all limb pair combinations was found (except for SD of CRP of the arms: $\chi_{[1,43]}^{2}=2.86, p=0.09$; and LALL: $\left.\chi_{[1,44]}^{2}=0.10, p=0.75\right)$, and revealed that coordinative stability increased when walking faster (SD of CRP of the legs: $\chi^{2}{ }_{[1,44]}=28.28, p<0.001$; MAML: $\chi^{2}{ }_{[1,43]}=9.32, p=0.002 ;$ LAML: $\chi^{2}[1,44]=20.02$, $p<0.001$; MALL: $\left.\chi^{2}{ }_{[1,43]}=21.14, p<0.001\right)$.

\subsection{Leading or lagging limb}

The number of participants that had a positive or negative sign of the MRP are presented in Table 2.

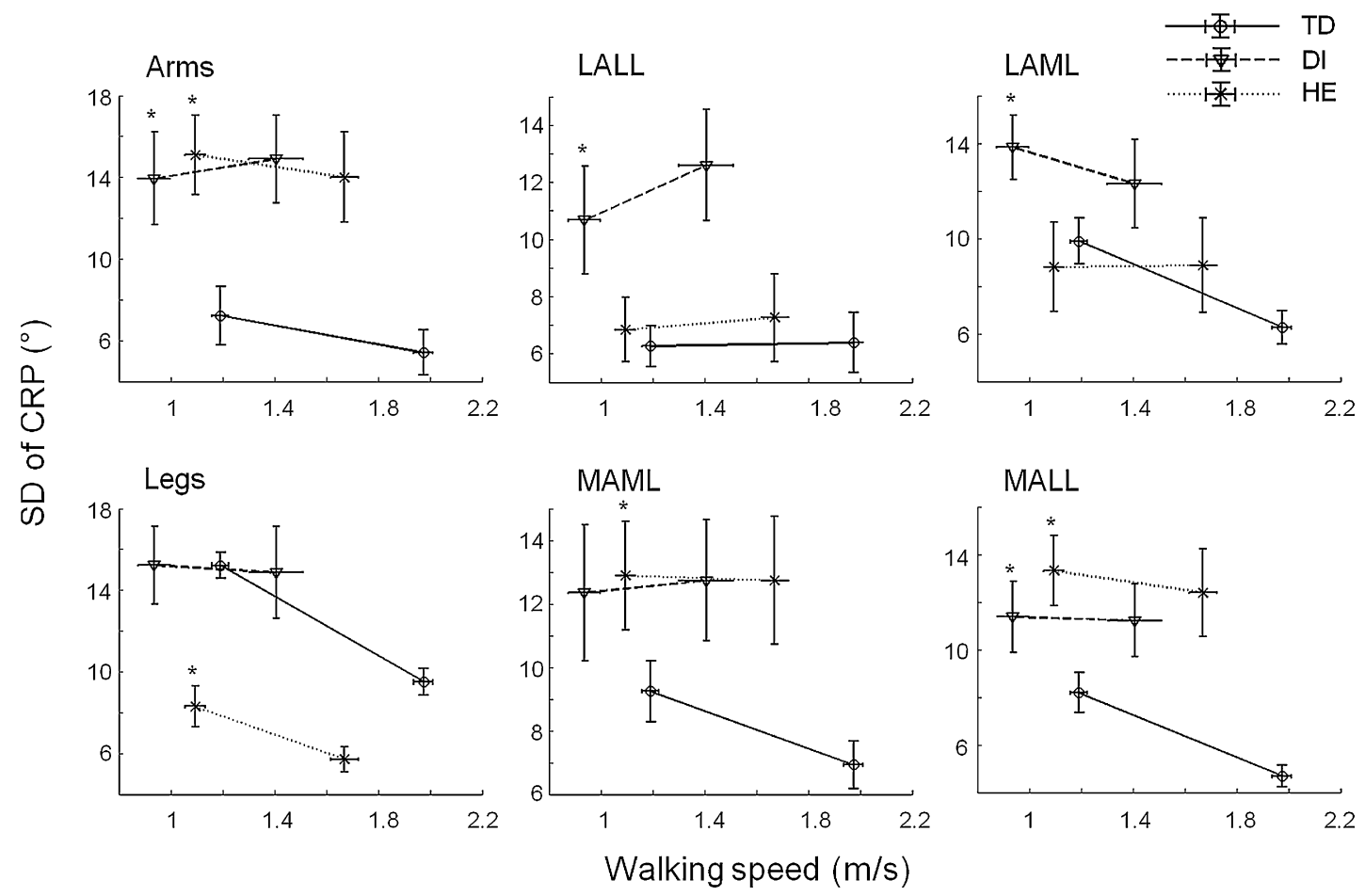

Fig. 4. Comparison of the level of coordinative stability between the three groups. Standard Deviation of the Continuous Relative Phase (SD of CRP) over the gait cycle is presented for the three groups. The symbols representing the groups are rounds for typically developing children (TD), triangles for children with diplegia (DI), and crosses for children with hemiplegia (HE). There are two symbols for each group, one for each walking speed condition. Additionally, the lines connecting the symbols are different for the three groups (full for TD, dashed for DI, and dotted for HE). Error bars represent the standard errors of the mean. Abbreviations: LALL = least affected arm-least affected leg, MAML = most affected arm-most affected leg, LAML = least affected arm-most affected leg, MALL = most affected arm-least affected leg. *Significantly different from TD (group effect). 
Table 2

Number of participants with a positive or negative sign of the Mean Relative Phase of each limb pair for the two walking conditions.

\begin{tabular}{|c|c|c|c|c|c|c|c|c|c|c|}
\hline & & \multicolumn{4}{|l|}{ FW } & \multicolumn{4}{|l|}{ FFW } & \multirow[t]{2}{*}{ Interpretation } \\
\hline & & $n$ positive & $n$ negative & $\chi^{2}[2, N]$ & $p$ & $n$ positive & $n$ negative & $\chi^{2}[2, N]$ & $p$ & \\
\hline \multirow[t]{6}{*}{ TD } & Arms & 14 & 10 & 0.336 & 0.414 & 13 & 10 & 0.196 & 0.532 & \\
\hline & Legs & 14 & 10 & 0.336 & 0.414 & 13 & 10 & 0.196 & 0.532 & \\
\hline & LALL & 20 & 4 & 6.000 & 0.001 & 19 & 4 & 5.473 & 0.002 & Leg ahead of arm. \\
\hline & MAML & 21 & 3 & 7.855 & $<\mathbf{0 . 0 0 1}$ & 21 & 2 & 9.462 & $<\mathbf{0 . 0 0 1}$ & Leg ahead of arm. \\
\hline & LAML & 4 & 20 & 6.000 & 0.001 & 3 & 20 & 7.276 & $<\mathbf{0 . 0 0 1}$ & Arm ahead of leg. \\
\hline & MALL & 3 & 21 & 7.855 & $<\mathbf{0 . 0 0 1}$ & 4 & 19 & 5.473 & $\mathbf{0 . 0 0 2}$ & Arm ahead of leg. \\
\hline \multirow[t]{6}{*}{ DI } & Arms & 7 & 7 & 0.000 & 1.000 & 7 & 8 & 0.033 & 0.796 & \\
\hline & Legs & 9 & 6 & 0.303 & 0.439 & 5 & 10 & 0.857 & 0.197 & \\
\hline & LALL & 4 & 11 & 1.727 & 0.071 & 10 & 5 & 0.857 & 0.197 & $\begin{array}{l}\text { Arm ahead of leg. Reversed } \\
\text { for fast walking. }\end{array}$ \\
\hline & MAML & 6 & 8 & 0.144 & 0.593 & 7 & 8 & 0.033 & 0.796 & \\
\hline & LAML & 11 & 4 & 1.727 & 0.071 & 8 & 7 & 0.033 & 0.796 & Leg ahead of arm. \\
\hline & MALL & 8 & 6 & 0.144 & 0.593 & 9 & 6 & 0.303 & 0.439 & \\
\hline \multirow[t]{6}{*}{$\mathrm{HE}$} & Arms & 2 & 8 & 1.978 & 0.054 & 5 & 6 & 0.046 & 0.763 & $\begin{array}{l}\text { Most affected arm ahead } \\
\text { of least affected arm. }\end{array}$ \\
\hline & Legs & 5 & 6 & 0.046 & 0.763 & 3 & 8 & 1.198 & 0.132 & \\
\hline & LALL & 10 & 1 & 4.422 & 0.007 & 10 & 1 & 4.422 & 0.007 & Leg ahead of arm. \\
\hline & MAML & 4 & 6 & 0.202 & 0.527 & 5 & 6 & 0.046 & 0.763 & \\
\hline & LAML & 1 & 10 & 4.422 & 0.007 & 1 & 10 & 4.422 & 0.007 & Arm ahead of leg. \\
\hline & MALL & 5 & 5 & 0.000 & 1.000 & 7 & 4 & 0.417 & 0.366 & \\
\hline
\end{tabular}

Note that the Relative Phase angle between the leg and arm segment was calculated as the phase angle of the leg minus the phase angle of the arm. Therefore a positive relative phase value indicates that the leg segment is ahead of the arm segment. The relative phase angle between the arms or between the legs was determined as the phase angle of the least affected limb minus the phase angle of the most affected limb. This means that a positive relative phase value reflects that the least affected limb is ahead of the most affected limb. For some groups, some participants were removed when all their trials showed an arm to leg frequency that was different from 1:1. The sample size of the $\chi^{2}$ test $(N)$ is the sum of the amount of positive numbers and the amount of negative numbers for that limb pair. Abbreviations: FW= preferred walking speed condition; FFW = "as fast as possible" walking speed condition; LALL= least affected arm-least affected leg; MAML = most affected arm-most affected leg; LAML = least affected arm-most affected leg; MALL = most affected armleast affected leg. Differences that were either borderline significant or significant are presented in bold.

For the coordination between arms and between legs, no limb was leading the movement in TD. However, in HE, the most affected arm was leading the least affected one at the lower walking speed (marginally significant).

For ipsilateral limb pairs, the leg normally leads the arm (TD), and this was similar in the least affected side of HE. However, no significant leading limb could be identified for HE on the most affected side, and for DI on both sides. Still, a trend for the least affected arm leading the leg was found for the lower walking speed in DI.

Considering contralateral limb pairs, the arm typically leads the leg (TD). Again, for HE this was similar in the least affected arm-most affected leg combination. However, such a clear leading limb could not be identified for the most affected arm-least affected leg combination for HE. For DI, no leading limb could be identified for either limb pair, although a trend for the most affected leg leading the least affected arm was found for the lower walking speed (in contrast to TD).

\section{Discussion}

The main result of the current study is that interlimb coordination during gait in children with CP differs significantly from that of TD children. Overall, children with CP show more 2:1 arm-to-leg swing ratio trials, altered phase relationships, decreased coordinative stability, and a different limb leading during walking compared to TD children.

Several children with hemiplegia showed a 2:1 arm-to-leg ratio for their most affected arm while the least affected arm showed a 1:1 arm-to-leg swing ratio (12/66 trials). To our knowledge this asymmetry in frequency coupling of the arms has not been previously described in children with $\mathrm{CP}$. Comparable results have been found in studies on stroke patients concerning the 2:1 arm-to-leg swing ratio on the hemiplegic side and a 1:1 ratio on the non-hemiplegic side (Ford, Wagenaar, \& Newell, 2007b; Wagenaar \& Van Emmerik, 1994). The 2:1 arm-to-leg ratios were not induced by the lower preferred walking speed in these children with $\mathrm{CP}$, because the 2:1 swing ratios persisted at the fast walking speed. This indicates that the most affected arm is responsible for the altered frequency coupling between arms and legs in children with hemiplegia (as described in hemiplegia after stroke). Additionally, we also found 2:1 arm-to-leg ratios in children with diplegia. Even though they showed this for both sides of the body, it did occur more frequently on the most affected side. In children with diplegia the legs are more affected than the arms (Dabney et al., 1997), but an asymmetric distribution of the affected limbs was often present. This might explain why the 2:1 arm-to-leg swing ratio occurred also in DI especially on the most affected side. Only few trials in TD children showed a similar asymmetry in arm-to-leg swing ratio. This can be explained by the results of a study by Wagenaar and van Emmerik (Wagenaar \& Van Emmerik, 1994), who described 'multistability' (i.e. 2:1 arm-to-leg frequency for an arm co-occurs with 1:1 arm-to-leg frequency of the other arm) which sometimes emerges at lower walking speeds for healthy adults. Apparently, in children with CP, this multistability occurs 
more frequently than in TD children. Additionally, in children with CP these two stable patterns emerging at the same time, persist at higher walking speeds.

In the trials where children with hemiplegia did show a 1:1 arm-to-leg ratio, they showed alterations in interlimb coordination, demonstrating the asymmetric distribution of the pathology. The arms and legs were both moving significantly less in anti-phase compared to TD children. Additionally, considering the other limb pair combinations, the alterations in interlimb coordination seemed to originate from the most affected arm. These results are in disagreement with findings from literature on adults with hemiplegia (post-stroke), where it was reported that stroke patients are able to coordinate their paretic and non-paretic sides equally well during treadmill walking (Ford, Wagenaar, \& Newell, 2007a; Stephenson et al., 2009). This may be due to a basic difference between stroke and CP (e.g. damage in the mature vs still developing brain) and/or the use of a treadmill in the stroke studies. Compared to overground walking, treadmill walking has been shown to induce a more consistent and symmetric gait pattern, accompanied by bilateral changes in the EMG activation patterns in hemiparetic stroke patients (Harris-Love, Forrester, Macko, Silver, \& Smith, 2001; Harris-Love, Macko, Whitall, \& Forrester, 2004). Although resulting in a less consistent gait pattern, overground walking more closely resembles actual walking in daily life compared to treadmill walking, and was therefore selected for this study.

Coordination of all limb pairs in children with diplegia was significantly different when compared to TD children when one of the legs was included (except between the most affected arm and least affected leg). Coordination between the arms in children with diplegia, on the other hand, did not differ from that of TD children. Therefore, the most affected limbs (i.e. legs) in children with diplegia seemed to limit the coordination capabilities of the limb couples. Thus, we propose that for both $\mathrm{CP}$ groups, the affected limbs rather than the compensating limbs were responsible for the modification of interlimb coordination. This is an important observation for gait rehabilitation, since it implies that training the affected limbs to increase swing amplitude during gait could improve interlimb coordination, and may therefore also improve quality of gait in children with CP. Future research should investigate how this observation may be translated into clinical advice.

Results on the stability of the coordination pattern (SD of CRP) mostly confirmed findings of the mean absolute relative phase (MARP). For children with hemiplegia, less stable coordination patterns were found whenever the most affected arm was included in the limb pair. This agrees with the literature on patients with hemiplegia after stroke (Garry, van Steenis, \& Summers, 2005; Wagenaar \& Van Emmerik, 1994), and confirms that the affected arm rather than the compensating arm constrains interlimb coordination. However, in the diplegic group, the compensating limbs (i.e. arms) influenced coordination stability, i.e. coordination between the arms was significantly more variable in children with diplegia. This is in agreement with results by Romkes et al. (2007), who found more variable kinematics of the upper limbs and trunk in children with diplegia while walking. The current results indicate that the less affected (i.e. compensating) limbs determined coordinative stability while the affected limbs modified the coordination pattern in children with diplegia. In children with diplegia, this could be due to the bilateral deficit of the lower limbs, which suggests that they will be more unstable during walking compared to children with hemiplegia. Furthermore there are more degrees of freedom for the arms compared to the legs during gait, which therefore can result in more movement variability in the arms than in the legs. It appears that the coordinative instability of the compensating limbs (i.e. arms) in children with diplegia may diminish when the affected limbs are assisted during gait or the movement has been trained. There would be less need for compensation under these circumstances.

With respect to the question which limb is leading in gait, we found that in TD children the leg led the movement when considering ipsilateral limb pairs, while the arm led the movement when considering the crossed limb pairs. At first sight, these results in TD children are in contrast to previous work in adults, where it was shown that maximal arm flexion (or extension) often precedes the contralateral (ipsilateral) heel strike (Murray, Sepic, \& Barnard, 1967) or contralateral maximal forward (ipsilateral backward) leg swing (Nanhoe-Mahabier et al., 2011). This difference might be due to the population studied (children vs adults) but also due to differences in calculating the phase difference. The studies mentioned used timing of only one anchor point (e.g. arm swing) during the gait cycle and not all data points of the gait cycle.

The pattern of limb-leading was different in children with CP. For children with diplegia, almost all limb pair coordination patterns were significantly different from TD. This was also reflected in the pattern of the limb-leading (either no leading limb could be identified or the limb leading phase was opposite compared to TD children). Similarly in children with hemiplegia, impaired coordination patterns in a limb pair were also reflected in opposite or no clear limb-leading within that limb pair (Arms, MAML and LALL). For children with diplegia the least affected arm led the least affected leg at the lower walking speed. This indicates that the least affected arm played a more active and prominent role in children with diplegia, thereby leading the leg movement. These results suggest that arm movements in children with CP may play a compensatory role for the affected legs during walking.

Overall, these results suggest that training or assisting the most affected limbs of $\mathrm{CP}$ children during walking to reach more normal patterns, can also decrease the compensations needed of the least affected limbs. This leads to visually more similar gait patterns between $\mathrm{CP}$ children and TD children, but possibly also to a higher quality of the gait pattern in children with CP.

\subsection{Functional implications: effects of speed}

Increasing walking speed had (almost) no effect on interlimb coordination in TD children, while it had more pronounced effects in $\mathrm{CP}$ children, especially in children with diplegia. For children with diplegia, increasing walking speed had clear 
beneficial effects on the mean absolute relative phase (MARP) for several limb pairs (LALL, LAML \& MAML), i.e. their coordination patterns approached those of TD children. Furthermore, when walking faster, coordination patterns became significantly more stable for most limb pair combinations. Lastly, as a result of the increase in speed, in more CP children the same limb (as in TD children) led the movement. This was the case for both children with diplegia (LALL \& LAML) and hemiplegia (arms). The current results of the beneficial influence of increased walking speed on interlimb coordination and coordinative stability are in agreement with findings in stroke patients (Kwakkel \& Wagenaar, 2002). These normalizing effects of increased walking speed on interlimb coordination have important implications for gait rehabilitation in children with CP. More specifically, these results indicate that allowing children with CP to exercise walking at higher walking speeds can improve gait rehabilitation in terms of improved interlimb coordination patterns. This appears to contradict recent results showing that gait asymmetries in children with CP are accentuated when running (Böhm \& Döderlein, 2012). We, however, examined two walking conditions in the current study whereas the previous study compared walking to running. The stabilizing effect of the increase in walking speed on interlimb coordination patterns has been reported for healthy adults (Donker, Beek, Wagenaar, \& Mulder, 2001; Wagenaar \& Van Emmerik, 2000), but not yet in children with CP. This stabilization with increasing speed is in contrast with one of the basic findings of studies on rhythmical bimanual coordination, where an increase in movement frequency affects the stability of an anti-phase pattern (Kelso, 1984; Swinnen, 2002). Differences between the task demands of walking and bimanual movement may account for this discrepancy (e.g. need to maintain balance during walking).

\subsection{Limitations}

Some limitations should be taken into account when interpreting the current results. Most of the children with $\mathrm{CP}$ had a GMFCS level 1. Accordingly, the CP group was only mildly affected. Hence the present results may underestimate the effects in a more general population of CP. Furthermore, interlimb coordination was determined from angular displacements of segments in the sagittal plane. This was based on the finding that the largest arm movements were present in the anteriorposterior direction in the currently evaluated children (Meyns et al., 2011). Therefore, we opted for a simplified kinematics approach (i.e. only sagittal plane), which was justified for the present patient group but this may be oversimplified for other more affected groups. Angle time series in human walking are not fully harmonic, which could have led to an overestimation of the standard deviation of relative phase. Specific parametric statistics have been used (i.e. Generalized Estimation Equations) that allowed walking speed to be included as a continuous independent variable (instead of a categorical one). This further limited the effects of differences in walking speed. Since a minority of the variables did not show a normal distribution, it could be argued that non-parametric tests should be used. A non-parametric equivalent of the generalized estimation equations, however, does not exist. Moreover, it has been shown that Generalized Estimation Equations are robust for non-Gaussian outcomes (Liang \& Zeger, 1986; Zeger \& Liang, 1986).

\section{Conclusions}

Interlimb coordination during walking is significantly altered in children with CP. Training or assisting the most affected limb movements in CP children during walking (to reach more normal coordination patterns), can lead to esthetically similar gait patterns between CP children and TD children, and to a higher quality of the CP gait pattern. Furthermore, increasing walking speed beneficially influences the coordination patterns (coordination, coordinative stability and limb-leading) of both CP groups, in particular children with diplegia, and may potentially be used as a rehabilitation strategy.

\section{Conflicting interests}

The authors declared no conflicts of interest with respect to the authorship and/or publication of this article.

\section{Acknowledgments}

This project was supported by a grant from 'bijzonder onderzoeksfonds' KU Leuven (OT/08/034). LVG received a PhD fellowship of the Research Foundation Flanders (FWO). JD was also supported by FWO (grant G.0901.11). SMB was funded by a visiting postdoctoral fellowship from the FWO (GP.030.10.N). Special thanks are due to the physical therapists of the Laboratory of Clinical Movement Analysis of the University Hospital Leuven (U.Z. Leuven).

\section{Appendix A. Supplementary data}

Supplementary data associated with this article can be found, in the online version, at doi:10.1016/j.ridd.2012.03.020.

\section{References}

Aisen, M. L., Kerkovich, D., Mast, J., Mulroy, S., Wren, T. A., Kay, R. M., et al. (2011). Cerebral palsy: Clinical care and neurological rehabilitation. Lancet Neurology, 10(9), 844-852. 
Behrman, A. L., \& Harkema, S. J. (2000). Locomotor training after human spinal cord injury: A series of case studies. Physical Therapy, 80(7), 688-700. Böhm, H., \& Döderlein, L. (2012). Gait asymmetries in children with cerebral palsy: Do they deteriorate with running? Gait $\mathcal{E}$ Posture, 35(2), 322-327.

Bruijn, S. M., Meyns, P., Jonkers, I., Kaat, D., \& Duysens, J. (2011). Control of angular momentum during walking in children with cerebral palsy. Research in Developmental Disabilities, 32(6), 2860-2866.

Dabney, K. W., Lipton, G. E., \& Miller, F. (1997). Cerebral palsy. Current Opinion in Pediatrics, 9(1), 81-88.

Davis, R. B., Ounpuu, S., Tyburski, D., \& Gage, J. R. (1991). A gait analysis data-collection and reduction technique. Human Movement Science, 10(5), 575-587.

Donker, S. F., \& Beek, P. J. (2002). Interlimb coordination in prosthetic walking: Effects of asymmetry and walking velocity. Acta Psychologica, 110(2-3), 265-288.

Donker, S. F., Beek, P. J., Wagenaar, R. C., \& Mulder, T. (2001). Coordination between arm and leg movements during locomotion. Journal of Motor Behavior, 33(1), $86-102$.

Fisher, N. I. (1993). Statistical analysis of circular data. Cambridge: Cambridge University Press.

Ford, M. P., Wagenaar, R. C., \& Newell, K. M. (2007a). Phase manipulation and walking in stroke. Journal of Neurologic Physical Therapy, 31(2), 85-91.

Ford, M. P., Wagenaar, R. C., \& Newell, K. M. (2007b). The effects of auditory rhythms and instruction on walking patterns in individuals post stroke. Gait $\mathcal{E}$ Posture, 26(1), 150-155.

Garry, M. I., van Steenis, R. E., \& Summers, J. J. (2005). Interlimb coordination following stroke. Human Movement Science, 24(5-6), 849-864.

Gutierrez, E. M., Bartonek, A., Haglund-Akerlind, Y., \& Saraste, H. (2003). Centre of mass motion during gait in persons with myelomeningocele. Gait E Posture, 18(2), 37-46

Harris-Love, M. L., Forrester, L. W., Macko, R. F., Silver, K. H., \& Smith, G. V. (2001). Hemiparetic gait parameters in overground versus treadmill walking. Neurorehabilitation and Neural Repair, 15(2), 105-112.

Harris-Love, M. L., Macko, R. F., Whitall, J., \& Forrester, L. W. (2004). Improved hemiparetic muscle activation in treadmill versus overground walking. Neurorehabilitation and Neural Repair, 18(3), 154-160.

Kadaba, M. P., Ramakrishnan, H. K., \& Wootten, M. E. (1990). Measurement of lower-extremity kinematics during level walking. Journal of Orthopaedic Research, $8(3), 383-392$.

Kelso, J. A. (1984). Phase transitions and critical behavior in human bimanual coordination. American Journal of Physiology, $246(6$ Pt 2), R1000-R1004.

Krasovsky, T., \& Levin, M. F. (2010). Review: Toward a better understanding of coordination in healthy and poststroke gait. Neurorehabilitation and Neural Repair, 24(3), 213-224.

Kwakkel, G., \& Wagenaar, R. C. (2002). Effect of duration of upper- and lower-extremity rehabilitation sessions and walking speed on recovery of interlimb coordination in hemiplegic gait. Physical Therapy, 82(5), 432-448.

Liang, K. Y., \& Zeger, S. L. (1986). Longitudinal data-analysis using generalized linear-models. Biometrika, 73, 13-22.

Meesen, R. L. J., Wenderoth, N., Temprado, J. J., Summers, J. J., \& Swinnen, S. P. (2006). The coalition of constraints during coordination of the ipsilateral and heterolateral limbs. Experimental Brain Research, 174(2), 367-375.

Meyns, P., Desloovere, K., Van Gestel, L., Massaad, F., Smits-Engelsman, B., \& Duysens, J. (2012). Altered arm posture in children with cerebral palsy is related to instability during walking. European Journal of Paediatric Neurology doi:10.1016/j.ejpn.2012.01.011 in press.

Meyns, P., Van Gestel, L., Massaad, F., Desloovere, K., Molenaers, G., \& Duysens, J. (2011). Arm swing during walking at different speeds in children with Cerebral Palsy and typically developing children. Research in Developmental Disabilities, 32(5), 1957-1964.

Milosevic, M., McConville, K. M., \& Masani, K. (2011). Arm movement improves performance in clinical balance and mobility tests. Gait E Posture, 33(3), 507-509.

Murray, M. P., Sepic, S. B., \& Barnard, E. J. (1967). Patterns of sagittal rotation of the upper limbs in walking. Physical Therapy, 47(4), 272-284.

Nanhoe-Mahabier, W., Snijders, A. H., Delval, A., Weerdesteyn, V., Duysens, J., Overeem, S., et al. (2011). Walking patterns in Parkinson's disease with and without freezing of gait. Neuroscience, 182, 217-224.

Palisano, R., Rosenbaum, P., Walter, S., Russell, D., Wood, E., \& Galuppi, B. (1997). Development and reliability of a system to classify gross motor function in children with cerebral palsy. Developmental Medicine and Child Neurology, 39(4), 214-223.

Romkes, J., Peeters, W., Oosterom, A. M., Molenaar, S., Bakels, I., \& Brunner, R. (2007). Evaluating upper body movements during gait in healthy children and children with diplegic cerebral palsy. Journal of Pediatric Orthopaedics - Part B, 16(3), 175-180.

Rose, J. (2009). Selective motor control in spastic cerebral palsy. Developmental Medicine and Child Neurology, 51(8), 578-579.

Sakamoto, M., Tazoe, T., Nakajima, T., Endoh, T., Shiozawa, S., \& Komiyama, T. (2007). Voluntary changes in leg cadence modulate arm cadence during simultaneous arm and leg cycling. Experimental Brain Research, 176(1), 188-192.

Stephenson, J. L., De Serres, S. J., \& Lamontagne, A. (2010). The effect of arm movements on the lower limb during gait after a stroke. Gait \& Posture, 31(1), 109-115

Stephenson, J. L., Lamontagne, A., \& De Serres, S. J. (2009). The coordination of upper and lower limb movements during gait in healthy and stroke individuals. Gait E' Posture, 29(1), 11-16.

Stergiou, N. (2004). Innovative analysis of human movement. Champaign, IL: Human Kinetics.

Swinnen, S. P. (2002). Intermanual coordination: From behavioural principles to neural-network interactions. Nature Reviews Neuroscience, 3(5), 350-361.

Swinnen, S. P., Jardin, K., Meulenbroek, R., Dounskaia, N., \& Hofkens-Van Den Brandt, M. (1997). Egocentric and allocentric constraints in the expression of patterns of interlimb coordination. Journal of Cognitive Neuroscience, 9(3), 348-377.

Van Emmerik, R. E., Wagenaar, R. C., \& Van Wegen, E. E. (1998). Interlimb coupling patterns in human locomotion: Are we bipeds or quadrupeds? Annals of the New York Academy of Sciences, 860, 539-542.

Wagenaar, R. C., \& Van Emmerik, R. E. A. (1994). Dynamics of pathological gait. Human Movement Science, 13(3-4), 441-471.

Wagenaar, R. C., \& Van Emmerik, R. E. A. (2000). Resonant frequencies of arms and legs identify different walking patterns. Journal of Biomechanics, 33(7), 853-861.

Woltring, H. J. (1986). A FORTRAN package for generalized, cross-validatory spline smoothing and differentiation. Advances in Engineering Software, 8(2), 104-113.

Zeger, S. L., \& Liang, K. Y. (1986). Longitudinal data analysis for discrete and continuous outcomes. Biometrics, 42, 121-130. 